\title{
Gender differences in waterpipe tobacco smoking among university students in four Eastern Mediterranean countries
}

\author{
Randah R. Hamadeh', Juhan Lee', Niveen M. E. Abu-Rmeileh ${ }^{3}$, Muhammad Darawad ${ }^{4}$, Aya Mostafa ${ }^{5}$, Khalid A. \\ Kheirallah ${ }^{6}$, Afzalhussein Yusufali ${ }^{7}$, Justin Thomas ${ }^{8}$, Mohamed Salama $^{9}$, Rima Nakkash ${ }^{10}$, Ramzi G. Salloum ${ }^{11}$
}

\begin{abstract}
INTRODUCTION Males have a higher prevalence of waterpipe tobacco smoking (WTS) than females in most Eastern Mediterranean Region (EMR) countries, with a smaller gender gap than that of cigarette smoking. The objective of this study was to determine gender differences among university students with respect to WTS initiation, smoking behavior, tobacco flavors, and expenditure on WTS, in four EMR countries.

METHODS A cross-sectional online survey was conducted based on convenient samples of ever waterpipe smokers among university students in four EMR countries (Egypt, Jordan, Occupied Palestinian Territories, and the United Arab Emirates) in 2016. The total samples included 2470 participants. Study participants were invited through flyers, university portals, emails and Facebook, followed by emails with links to the internet survey. RESULTS Females $(80.4 \%)$ were more likely than males $(66.4 \%, \mathrm{p}<0.001)$ to be in the younger age group (18-22 years) and they were less likely to be current waterpipe smokers (females, $60.0 \%$; males $69.5 \%$, p<0.001). Twothirds of students across both genders smoked their first waterpipe at the age of 15-19 years, with more females starting with family members. Over one-third of males and $14.9 \%$ of the females usually smoked $\geq 10$ heads $(p<0.001)$. About half $(46.6 \%)$ of females smoked for less than half an hour compared to $30.5 \%$ of males $(\mathrm{p}<0.001)$. Only $1 \%$ of females smoked non-flavored tobacco compared to $11 \%$ of males $(\mathrm{p}<0.001)$. There was a significant $(\mathrm{p}=0.05)$ positive correlation $(\mathrm{r}=0.808)$ with respect to tobacco flavor usually smoked between males and females with apple/double apple being the most popular.
\end{abstract}

CONCLUSIONS There were gender differences in WTS in several aspects. The study has implications for educational establishments, tobacco control and women civil society groups, as well as policymakers.

\author{
AFFILIATION \\ 1 College of Medicine and Medical \\ Sciences, Arabian Gulf University, \\ Manama, Bahrain \\ 2 Department of Health Education and \\ Behavior, College of Health and Human \\ Performance, University of Florida, \\ Gainesville, United States \\ 3 Institute of Community and Public \\ Health, Birzeit University, Birzeit, \\ Occupied Palestinian Territories \\ 4 School of Nursing, The University of \\ Jordan, Amman, Jordan \\ 5 Department of Community, \\ Environmental and Occupational \\ Medicine, Ain Shams University, Cairo, \\ Egypt \\ 6 Department of Public Health, Jordan \\ University of Science and Technology, \\ Irbid, Jordan \\ 7 Hatta Hospital, Dubai Medical \\ College, Dubai, United Arab Emirates \\ 8 Department of Psychology, Zayed \\ University, Abu Dhabi, United Arab \\ Emirates \\ 9 Institute of Global Health and \\ Human Ecology, The American \\ University in Cairo, New Cairo, Egypt \\ 10 Faculty of Health Sciences, \\ American University of Beirut, Beirut, \\ Lebanon \\ 11 Department of Health Outcomes \\ and Biomedical Informatics, College \\ of Medicine, University of Florida, \\ Gainesville, United States \\ CORRESPONDENCE TO \\ Randah R. Hamadeh. College of \\ Medicine and Medical Sciences, \\ Arabian Gulf University, Manama, \\ Bahrain.E-mail: randah@agu.edu.bh \\ ORCID ID: https://orcid.org/0000-0002- \\ 4205-1354 \\ KEYWORDS \\ waterpipe, shisha, hookah, youth, EMR
}

Received: 26 July 2020

Revised: 27 October 2020

Accepted: 29 October 2020 


\section{INTRODUCTION}

The prevalence of waterpipe tobacco smoking (WTS) in the Eastern Mediterranean Region (EMR) is on the rise especially among youth ${ }^{1}$. Of the 25 countries that assessed WTS in the Global Youth Tobacco Survey among students aged 13-15 years, Lebanon (36.9\%) and the West Bank (32.7\%) had the highest prevalence rates ${ }^{1}$. Although WTS prevalence is higher in males in most EMR countries, the gender gap is much smaller than that of cigarette smoking ${ }^{2,3}$. In a number of countries in the EMR, restrictions towards smoking are religious, cultural and social, and are more firmly applied to females than males, and to cigarette smoking compared with WTS ${ }^{4}$. Recent evidence from Egypt indicates that the gender gap for certain tobacco product types is narrowing and even higher among females for electronic nicotine delivery systems (ENDS) use ${ }^{5}$. Previous studies have attributed this finding partially to the social acceptability of noncigarette tobacco products compared with cigarettes ${ }^{6-8}$. Further, the perception of reduced harm and the availability of flavored waterpipe tobacco are among the reasons for its social acceptability ${ }^{8}$. It is common for women in the EMR to report WTS initiation within their families ${ }^{8,9}$. In Turkey, the presence of waterpipe smokers among family members was found to have significant effects on the prevalence rate of WTS amongst university students ${ }^{10}$.

Flavored waterpipe tobacco ('maassel') is the most preferred tobacco by waterpipe smokers globally, particularly among women ${ }^{11,12}$. In young people, flavors are reported as a common reason to experiment with WTS ${ }^{9}$. The World Health Organization (WHO) warned that women and girls are a major target for the tobacco industry to recruit new users and the rise in smoking among women has been associated with industry promotion of flavored tobacco ${ }^{13}$.

Given that there have not been sufficient studies comparing the behaviors of men and women in the EMR with respect to WTS, the objective of this study was to determine if there were gender differences among university students with respect to WTS initiation, smoking behavior, waterpipe tobacco flavors, and expenditure on WTS, in four EMR countries.

\section{METHODS}

A cross-sectional online survey was conducted based on convenience samples of ever waterpipe smokers among university students in four EMR countries: Egypt (2 universities), Jordan (2 universities), Occupied Palestinian Territories (OPT, 1 university), and the United Arab Emirates (UAE, 2 universities), between April and December 2016. The two universities from Egypt had an enrolment of 91041 and 168970, those of Jordan 20000 and 37692, and those of the UAE 500 and 9217, and 13963 for the $\mathrm{OPT}^{14}$. An estimated sample size of 384 students from each country was computed based on a $50 \%$ prevalence of WTS and a 95\% confidence interval. We targeted a sample of 400 participants per site to ensure adequate power for another component of this survey, reported elsewhere ${ }^{14,15}$.

We recruited student samples that were heterogeneous with respect to gender and study level (undergraduate vs graduate). Participants were initially invited through flyers, university portals, emails and Facebook. Students then received emails with links to the internet survey available in both Arabic and English and they were assured that their responses would remain anonymous. Participants were further assured that their smoking status would not be revealed and that they could leave the survey at any time. Financial incentives were not provided for participation in the survey. The study protocol was approved by the institutional review boards of all participating institutions. All study participants had to consent before taking the survey. The original dataset used and analyzed during the current study is available from an author (RGS), on reasonable request.

The inclusion criteria were age ( $\geq 18$ years), currently enrolled in the university or will be in the next year, and had ever smoked waterpipe for at least one or two puffs. More details on the methods of student recruitment at the participating universities are mentioned elsewhere ${ }^{14-16}$. Students who were not eligible or did not complete the online consent form were excluded.

The questionnaire was based on a standard survey on WTS and included demographic characteristics of waterpipe smokers, WTS history, WTS initiation, current use of waterpipe including flavors, in addition to concurrent use of other tobacco products. Current waterpipe smokers and current cigarette smokers were defined as those who smoked in the last 30 
days prior to the survey. Students who smoked the waterpipe the day of the survey or the previous day were considered daily smokers.

We compared males and females in all countries by: sociodemographic characteristics; tobacco use status within last 30 days by type; waterpipe smoking initiation by age, person(s) and place; most recent WTS session by duration of smoking; number of waterpipe heads, and place smoked; usual persons smoking waterpipe with, and places of smoking and purchase; and the top five waterpipe flavors usually smoked. Means and standard deviations were calculated for continuous variables and independent sample t-test was applied. SPSS version 22 was used for conducting the statistical analysis. Chi-squared test was used to determine gender differences within each country and overall, for the categorical variables. Spearman correlation (r) was calculated for the ranks of waterpipe flavors between genders. Differences were considered significant for $\mathrm{p}$-value $<0.05$.

\section{RESULTS}

The number of the university students that participated in the study was 2470 across the 4 countries. There were 728 respondents from Egypt (27.5\% females), 790 from Jordan (37.1\% females), 772 from the OPT ( $52.1 \%$ females), and 180 from the UAE ( $42.8 \%$ females).

\section{Demographic characteristics}

There were statistically significant differences between females and males with respect to age, educational level, marital status and employment, in the total sample (Table 1). More female students $(80.4 \%)$ were in the younger age group (18-22 years) than male students $(66.4 \%)(\mathrm{p}<0.001)$. Bachelor's, non-medical degree, was the highest level attained by female $(84.0 \%)$ and male $(72.3 \%)$ students $(p<0.001)$ with equal percentages in the OPT but higher among females in the other countries. More females were unmarried than males with the sample from Egypt having the lowest proportions of unmarried across both genders. The gender gap in employment status was high in Jordan, the OPT, and in the overall sample $(\mathrm{p}<0.001)$, with more males being employed than females. Egypt had the highest employment among its female and male students. The majority of male $(92.7 \%)$ and female (93.9\%) students were citizens of their respective countries of residence.

Table 1. Demographic characteristics of study participants in four EMR countries, 2016 (N=2470)

\begin{tabular}{|c|c|c|c|c|c|c|c|c|c|c|}
\hline \multirow[t]{2}{*}{ Characteristics } & \multicolumn{2}{|c|}{ Jordan } & \multicolumn{2}{|c|}{ OPT } & \multicolumn{2}{|c|}{ UAE } & \multicolumn{2}{|c|}{ Egypt } & \multicolumn{2}{|c|}{ Total } \\
\hline & $\begin{array}{l}\text { Female } \\
n(\%)\end{array}$ & $\begin{array}{l}\text { Male } \\
n(\%)\end{array}$ & $\begin{array}{c}\text { Female } \\
n(\%)\end{array}$ & $\begin{array}{l}\text { Male } \\
n(\%)\end{array}$ & $\begin{array}{l}\text { Female } \\
n(\%)\end{array}$ & $\begin{array}{l}\text { Male } \\
n(\%)\end{array}$ & $\begin{array}{c}\text { Female } \\
n(\%)\end{array}$ & $\begin{array}{l}\text { Male } \\
n(\%)\end{array}$ & $\begin{array}{c}\text { Female } \\
n(\%)\end{array}$ & $\begin{array}{l}\text { Male } \\
n(\%)\end{array}$ \\
\hline \multicolumn{11}{|l|}{ Age (years) } \\
\hline $18-22$ & 229 (81.2) & 393 (80.7) & 346 (88.9) & $303(83.0)$ & $60(78.9)$ & $82(83.7)$ & $20(29.4)$ & $203(38.4)$ & 655 (80.4) & 981 (66.4) \\
\hline$\geq 23$ & 53 (18.8) & 94 (19.3) & 43 (11.1) & $62(17.0)$ & $16(21.1)$ & 16 (16.3) & 48 (70.6) & $325(61.6)$ & $160(19.6)$ & 497 (33.6) \\
\hline Total & 282 & 487 & 389 & 365 & 76 & 98 & 68 & 528 & 815 & 1478 \\
\hline$p$-value* & \multicolumn{2}{|c|}{0.863} & \multicolumn{2}{|c|}{0.019} & \multicolumn{2}{|c|}{0.425} & \multicolumn{2}{|c|}{0.147} & \multicolumn{2}{|c|}{$<0.001$} \\
\hline \multicolumn{11}{|l|}{ Degree level } \\
\hline Diploma & $17(5.9)$ & $73(14.9)$ & $10(2.6)$ & $9(2.5)$ & $4(5.2)$ & 16 (15.8) & $3(4.9)$ & 59 (12.1) & $34(4.2)$ & 157 (11.0) \\
\hline $\begin{array}{l}\text { Bachelor's, } \\
\text { non-medical }\end{array}$ & 236 (81.7) & $323(66.1)$ & 340 (89.5) & 316 (89.3) & 60 (77.9) & 73 (72.3) & $42(68.9)$ & $324(66.4)$ & $678(84.0)$ & 1036 (72.3) \\
\hline Medical & $29(10.0)$ & 78 (16.0) & $1(0.3)$ & $3(0.8)$ & $9(11.7)$ & $7(6.9)$ & $5(8.2)$ & $32(6.6)$ & $44(5.5)$ & $120(8.4)$ \\
\hline Graduate & $7(2.4)$ & $15(3.0)$ & $29(7.6)$ & $26(7.3)$ & $4(5.2)$ & $5(5.0)$ & 11 (18.0) & 73 (15.0) & $51(6.3)$ & 119 (8.3) \\
\hline Total & 289 & 489 & 380 & 354 & 77 & 101 & 61 & 488 & 807 & 1432 \\
\hline$p$-value & \multicolumn{2}{|c|}{$<0.001$} & \multicolumn{2}{|c|}{0.759} & \multicolumn{2}{|c|}{0.127} & \multicolumn{2}{|c|}{0.381} & \multicolumn{2}{|c|}{$<0.001$} \\
\hline \multicolumn{11}{|l|}{ Marital status } \\
\hline Never married & 283 (96.9) & $479(96.6)$ & 364 (93.3) & 355 (96.7) & 74 (96.1) & $97(95.1)$ & 57 (83.8) & 403 (77.4) & 778 (94.1) & 1334 (89.8) \\
\hline Ever married & $9(3.1)$ & $17(3.4)$ & $26(6.7)$ & $12(3.3)$ & $3(3.9)$ & $5(4.9)$ & 11 (16.2) & $118(22.6)$ & $49(5.9)$ & $152(10.2)$ \\
\hline Total & 292 & 496 & 390 & 367 & 77 & 102 & 68 & 521 & 827 & 1486 \\
\hline p-value & \multicolumn{2}{|c|}{0.793} & \multicolumn{2}{|c|}{0.032} & \multicolumn{2}{|c|}{0.747} & \multicolumn{2}{|c|}{0.225} & \multicolumn{2}{|c|}{$<0.001$} \\
\hline
\end{tabular}


Table 1. Continued

\begin{tabular}{|c|c|c|c|c|c|c|c|c|c|c|}
\hline \multirow[t]{2}{*}{ Characteristics } & \multicolumn{2}{|c|}{ Jordan } & \multicolumn{2}{|c|}{ OPT } & \multicolumn{2}{|c|}{ UAE } & \multicolumn{2}{|c|}{ Egypt } & \multicolumn{2}{|c|}{ Total } \\
\hline & $\begin{array}{c}\text { Female } \\
n(\%)\end{array}$ & $\begin{array}{l}\text { Male } \\
n(\%)\end{array}$ & $\begin{array}{c}\text { Female } \\
n(\%)\end{array}$ & $\begin{array}{l}\text { Male } \\
n(\%)\end{array}$ & $\begin{array}{c}\text { Female } \\
n(\%)\end{array}$ & $\begin{array}{l}\text { Male } \\
n(\%)\end{array}$ & $\begin{array}{c}\text { Female } \\
n(\%)\end{array}$ & $\begin{array}{l}\text { Male } \\
n(\%)\end{array}$ & $\begin{array}{c}\text { Female } \\
n(\%)\end{array}$ & $\begin{array}{l}\text { Male } \\
n(\%)\end{array}$ \\
\hline \multicolumn{11}{|l|}{$\begin{array}{l}\text { Employment } \\
\text { status }\end{array}$} \\
\hline Unemployed & $225(82.1)$ & $315(68.8)$ & $271(78.1)$ & $196(59.6)$ & 60 (78.9) & 71 (71.7) & $24(41.4)$ & $200(41.9)$ & $580(76.8)$ & 782 (57.4) \\
\hline Employed & $49(17.9)$ & $143(31.2)$ & 76 (21.9) & $133(40.4)$ & $16(21.1)$ & $28(28.3)$ & $34(58.6)$ & $277(58.1)$ & 175 (23.2) & $581(42.6)$ \\
\hline Total & 274 & 458 & 347 & 329 & 76 & 99 & 58 & 477 & 755 & 1363 \\
\hline p-value & \multicolumn{2}{|c|}{$<0.001$} & \multicolumn{2}{|c|}{$<0.001$} & \multicolumn{2}{|c|}{0.274} & \multicolumn{2}{|c|}{0.936} & \multicolumn{2}{|c|}{$<0.001$} \\
\hline \multicolumn{11}{|l|}{$\begin{array}{l}\text { Citizenship } \\
\text { status }\end{array}$} \\
\hline Citizen & 267 (91.8) & $434(88.0)$ & 387 (99.5) & 366 (99.2) & $53(68.8)$ & $58(56.9)$ & $68(100.0)$ & 522 (99.4) & 775 (93.9) & 1380 (92.7) \\
\hline $\begin{array}{l}\text { Non-citizen/ } \\
\text { expatriate }\end{array}$ & $24(8.2)$ & 59 12.0) & $2(0.5)$ & $3(0.8)$ & 24 (31.2) & $44(43.1)$ & $0(0.0)$ & $3(0.6)$ & $50(6.1)$ & $109(7.3)$ \\
\hline Total & 291 & 493 & 389 & 369 & 77 & 102 & 68 & 525 & 825 & 1489 \\
\hline p-value & \multicolumn{2}{|c|}{0.102} & \multicolumn{2}{|c|}{0.611} & \multicolumn{2}{|c|}{0.102} & \multicolumn{2}{|c|}{0.532} & \multicolumn{2}{|c|}{0.251} \\
\hline
\end{tabular}

OPT: Occupied Palestinian Territories. UAE: United Arab Emirates. The numbers vary by each variable due to missing values. ${ }^{*} \mathrm{p}$-values for all characteristics were derived from chi-squared tests.

\section{Current waterpipe and other types of tobacco smoking}

There were more current smokers of any type of tobacco among males $(84.9 \%)$ than females $(66.2 \%)$ in the overall sample $(\mathrm{p}<0.001)$ and within each country, with the smallest gender gap in Egypt.

The UAE had the lowest proportion of current waterpipe smokers among females (49.4\%) and males (51.5\%). Egypt had the largest gender gap in current WTS (males $58.7 \%$; females $77.2 \%$ ).
All female waterpipe smokers in Egypt and the UAE were non-daily smokers (Table 2). Electronic waterpipe smoking among males (11.9\%) was almost double that of their female counterparts $(6.6 \%)$ with the highest difference observed in the UAE.

Current cigarette smoking in males $(50.3 \%)$ was double that of females $(19.4 \%)$ in the total sample $(p<0.001)$ and in all countries, with the exception of Egypt. Female students from Egypt had the highest prevalence of cigarette smoking and the smallest

Table 2. Tobacco use status (within last 30 days) by type in four EMR countries, 2016 ( $N=2470$ )

\begin{tabular}{|c|c|c|c|c|c|c|c|c|c|c|}
\hline \multirow[t]{2}{*}{ Tobacco use } & \multicolumn{2}{|c|}{ Jordan } & \multicolumn{2}{|c|}{ OPT } & \multicolumn{2}{|c|}{ UAE } & \multicolumn{2}{|c|}{ Egypt } & \multicolumn{2}{|c|}{ Total } \\
\hline & $\begin{array}{c}\text { Female } \\
n(\%)\end{array}$ & $\begin{array}{l}\text { Male } \\
n(\%)\end{array}$ & $\begin{array}{c}\text { Female } \\
n(\%)\end{array}$ & $\begin{array}{l}\text { Male } \\
n(\%)\end{array}$ & $\begin{array}{c}\text { Female } \\
n(\%)\end{array}$ & $\begin{array}{l}\text { Male } \\
n(\%)\end{array}$ & $\begin{array}{c}\text { Female } \\
n(\%)\end{array}$ & $\begin{array}{l}\text { Male } \\
n(\%)\end{array}$ & $\begin{array}{c}\text { Female } \\
n(\%)\end{array}$ & $\begin{array}{l}\text { Male } \\
n(\%)\end{array}$ \\
\hline \multicolumn{11}{|l|}{ Any tobacco } \\
\hline Current user & $203(70.5)$ & 412 (84.1) & $244(63.0)$ & 301 (82.0) & 40 (51.9) & 81 (81.0) & $54(83.1)$ & 451 (88.6) & $541(66.2)$ & $1245(84.9)$ \\
\hline Former user & 85 (29.5) & 78 (15.9) & $143(37.0)$ & $66(18.0)$ & $37(48.1)$ & $19(19.0)$ & 11 (16.9) & $58(11.4)$ & 276 (33.8) & $221(15.1)$ \\
\hline Total & 288 & 490 & 387 & 367 & 77 & 100 & 65 & 509 & 817 & 1466 \\
\hline$p$-value $e^{*}$ & \multicolumn{2}{|c|}{$<0.001$} & \multicolumn{2}{|c|}{$<0.001$} & \multicolumn{2}{|c|}{$<0.001$} & \multicolumn{2}{|c|}{0.197} & \multicolumn{2}{|c|}{$<0.001$} \\
\hline \multicolumn{11}{|l|}{ Waterpipe } \\
\hline $\begin{array}{l}\text { Current, non- } \\
\text { daily smoker }\end{array}$ & 171 (59.8) & $279(58.2)$ & $215(55.7)$ & $200(55.2)$ & $38(49.4)$ & 51 (51.5) & $37(58.7)$ & $368(74.8)$ & 461 (56.8) & $898(62.7)$ \\
\hline $\begin{array}{l}\text { Current, daily } \\
\text { smoker }\end{array}$ & $14(4.9)$ & 54 (11.3) & $12(3.1)$ & $43(11.9)$ & $0(0.0)$ & $2(2.0)$ & $0(0.0)$ & $12(2.4)$ & $26(3.2)$ & $111(7.8)$ \\
\hline Former smoker & 101 (35.3) & $146(30.5)$ & $159(41.2)$ & 119 (32.9) & $39(50.6)$ & $46(46.5)$ & 26 (41.3) & 112 (22.8) & $325(40.0)$ & 423 (29.5) \\
\hline Total & 286 & 479 & 386 & 362 & 77 & 99 & 63 & 492 & 812 & 1432 \\
\hline$p$-value & \multicolumn{2}{|c|}{0.008} & \multicolumn{2}{|c|}{$<0.001$} & \multicolumn{2}{|c|}{0.416} & \multicolumn{2}{|c|}{0.004} & \multicolumn{2}{|c|}{$<0.001$} \\
\hline
\end{tabular}


Table 2. Continued

\begin{tabular}{|c|c|c|c|c|c|c|c|c|c|c|}
\hline \multirow[t]{2}{*}{ Tobacco use } & \multicolumn{2}{|c|}{ Jordan } & \multicolumn{2}{|c|}{ OPT } & \multicolumn{2}{|c|}{ UAE } & \multicolumn{2}{|c|}{ Egypt } & \multicolumn{2}{|c|}{ Total } \\
\hline & $\begin{array}{c}\text { Female } \\
n(\%)\end{array}$ & $\begin{array}{l}\text { Male } \\
n(\%)\end{array}$ & $\begin{array}{c}\text { Female } \\
n(\%)\end{array}$ & $\begin{array}{l}\text { Male } \\
n(\%)\end{array}$ & $\begin{array}{l}\text { Female } \\
n(\%)\end{array}$ & $\begin{array}{l}\text { Male } \\
n(\%)\end{array}$ & $\begin{array}{l}\text { Female } \\
n(\%)\end{array}$ & $\begin{array}{l}\text { Male } \\
n(\%)\end{array}$ & $\begin{array}{l}\text { Female } \\
n(\%)\end{array}$ & $\begin{array}{l}\text { Male } \\
n(\%)\end{array}$ \\
\hline \multicolumn{11}{|l|}{$\begin{array}{l}\text { Electronic } \\
\text { waterpipe } \\
\text { (e-hookah) }\end{array}$} \\
\hline Current user & $28(9.9)$ & $52(11.0)$ & $13(3.4)$ & $7(2.0)$ & $3(3.9)$ & 18 (18.2) & $9(14.8)$ & 89 (18.9) & $53(6.6)$ & 166 (11.9) \\
\hline Former user & $25(8.8)$ & $58(12.3)$ & $7(1.8)$ & $10(2.8)$ & $21(27.6)$ & 16 (16.2) & 10 (16.4) & $45(9.5)$ & $63(7.9)$ & $129(9.2)$ \\
\hline Never user & 231 (81.3) & 363 (76.7) & 360 (94.7) & 335 (95.2) & $52(68.4)$ & 65 (65.7) & $42(68.9)$ & 338 (71.6) & $685(85.5)$ & 1101 (78.9) \\
\hline Total & 284 & 473 & 380 & 352 & 76 & 99 & 61 & 472 & 801 & 1396 \\
\hline p-value & \multicolumn{2}{|c|}{0.268} & \multicolumn{2}{|c|}{0.339} & \multicolumn{2}{|c|}{0.007} & \multicolumn{2}{|c|}{0.221} & \multicolumn{2}{|c|}{$<0.001$} \\
\hline \multicolumn{11}{|l|}{ Cigarettes } \\
\hline Current smoker & 55 (19.2) & $230(47.3)$ & 57 (15.1) & $168(46.7)$ & $16(21.1)$ & $55(55.0)$ & $27(47.4)$ & $263(55.1)$ & $155(19.4)$ & 716 (50.3) \\
\hline Former smoker & $27(9.4)$ & $40(8.2)$ & $25(6.6)$ & $12(3.3)$ & 15 (19.7) & $9(9.0)$ & $0(0.0)$ & $20(4.2)$ & $67(8.4)$ & $81(5.7)$ \\
\hline Never smoker & 204 (71.3) & $216(44.4)$ & $296(78.3)$ & $180(50.0)$ & $45(59.2)$ & $36(36.0)$ & $30(52.6)$ & $194(40.7)$ & 575 (72.1) & $626(44.0)$ \\
\hline Total & 286 & 486 & 378 & 360 & 76 & 100 & 57 & 477 & 797 & 1423 \\
\hline$p$-value & \multicolumn{2}{|c|}{$<0.001$} & \multicolumn{2}{|c|}{$<0.001$} & \multicolumn{2}{|c|}{$<0.001$} & \multicolumn{2}{|c|}{0.096} & \multicolumn{2}{|c|}{$<0.001$} \\
\hline \multicolumn{11}{|l|}{$\begin{array}{l}\text { Electronic } \\
\text { cigarettes } \\
\text { (e-cigarettes / } \\
\text { vape pens) }\end{array}$} \\
\hline Current user & $17(6.0)$ & $60(12.5)$ & $2(0.5)$ & $13(3.7)$ & $2(2.6)$ & 18 (18.2) & $11(18.6)$ & $63(13.5)$ & $32(4.0)$ & $154(11.0)$ \\
\hline Former user & $24(8.4)$ & $62(12.9)$ & $15(4.0)$ & $17(4.8)$ & $20(26.0)$ & 18 (18.2) & $2(3.4)$ & $42(9.0)$ & $61(7.6)$ & 139 (9.9) \\
\hline Never user & 244 (85.6) & 359 (74.6) & 360 (95.5) & 321 (91.5) & 55 (71.4) & $63(63.6)$ & $46(78.0)$ & $362(77.5)$ & 705 (88.3) & 1105 (79.0) \\
\hline Total & 285 & 481 & 377 & 351 & 77 & 99 & 59 & 467 & 798 & 1398 \\
\hline p-value & \multicolumn{2}{|c|}{0.001} & \multicolumn{2}{|c|}{0.009} & \multicolumn{2}{|c|}{0.004} & & 28 & & 001 \\
\hline Cigar & & & & & & & & & & \\
\hline Current smoker & $21(7.4)$ & $69(14.5)$ & $4(1.1)$ & $38(10.7)$ & $2(2.6)$ & $12(12.1)$ & $9(15.8)$ & $54(11.6)$ & $36(4.5)$ & $173(12.4)$ \\
\hline Former smoker & $18(6.4)$ & 64 (13.4) & $16(4.2)$ & 41 (11.6) & $6(7.9)$ & 18 (18.2) & $5(8.8)$ & $35(7.5)$ & $45(5.7)$ & 158 (11.3) \\
\hline Never smoker & 244 (86.2) & $343(72.1)$ & $359(94.7)$ & 275 (77.7) & 68 (89.5) & 69 (69.7) & $43(75.4)$ & 376 (80.9) & 714 (89.8) & 1063 (76.3) \\
\hline Total & 283 & 476 & 379 & 354 & 76 & 99 & 57 & 465 & 795 & 1394 \\
\hline$p$-value & & 001 & & 001 & & & 0.6 & 00 & & 001 \\
\hline Regular pipe & & & & & & & & & & \\
\hline Current smoker & $24(8.5)$ & $36(7.5)$ & $0(0.0)$ & $5(1.4)$ & $6(7.9)$ & $10(10.2)$ & $3(4.8)$ & $33(7.1)$ & $33(4.1)$ & $84(6.0)$ \\
\hline Former smoker & $9(3.2)$ & $37(7.8)$ & $7(1.8)$ & $14(4.0)$ & 12 (15.8) & 15 (15.3) & $3(4.8)$ & $22(4.7)$ & $31(3.9)$ & $88(6.3)$ \\
\hline Never smoker & 250 (88.3) & $404(84.7)$ & 372 (98.2) & 333 (94.6) & $58(76.3)$ & 73 (74.5) & $56(90.3)$ & 413 (88.2) & 736 (92.0) & $1223(87.7)$ \\
\hline Total & 283 & 477 & 379 & 352 & 76 & 98 & 62 & 468 & 800 & 1395 \\
\hline$p$-value & & 37 & & 14 & & & 0.8 & 09 & & 007 \\
\hline Midwakh & & & & & & & & & & \\
\hline Current smoker & $20(7.1)$ & 65 (13.7) & $0(0.0)$ & $8(2.3)$ & 10 (13.2) & $52(52.0)$ & $4(6.6)$ & $34(7.2)$ & $34(4.3)$ & 159 (11.4) \\
\hline Former smoker & $9(3.2)$ & $46(9.7)$ & $1(0.3)$ & $8(2.3)$ & $12(15.8)$ & $6(6.0)$ & $2(3.3)$ & $24(5.1)$ & $24(3.0)$ & $84(6.0)$ \\
\hline Never smoker & 254 (89.8) & 363 (76.6) & 377 (99.7) & 334 (95.4) & 54 (71.1) & $42(42.0)$ & 55 (90.2) & 416 (87.8) & 740 (92.7) & 1155 (82.6) \\
\hline Total & 283 & 474 & 378 & 350 & 76 & 100 & 61 & 474 & 798 & 1398 \\
\hline p-value & & 001 & & 01 & & & 0.8 & 11 & & 001 \\
\hline $\begin{array}{l}\text { Smokeless } \\
\text { tobacco }\end{array}$ & & & & & & & & & & \\
\hline Current user & $21(7.4)$ & $28(5.9)$ & $2(0.5)$ & $4(1.1)$ & $1(1.3)$ & $9(9.4)$ & $3(5.4)$ & $31(6.7)$ & $27(3.4)$ & $72(5.2)$ \\
\hline Former user & $4(1.4)$ & $24(5.1)$ & $3(0.8)$ & $5(1.4)$ & $2(2.6)$ & 7 (7.3) & $4(7.1)$ & $22(4.8)$ & $13(1.6)$ & $58(4.2)$ \\
\hline Never user & 259 (91.2) & $422(89.0)$ & 376 (98.7) & $343(97.4)$ & 73 (96.1) & 80 (83.3) & 49 (87.5) & $407(88.5)$ & $757(95.0)$ & $1252(90.6)$ \\
\hline Total & 284 & 474 & 381 & 352 & 76 & 96 & 56 & 460 & 797 & 1382 \\
\hline p-value & & & & 64 & & & & 04 & & 001 \\
\hline
\end{tabular}

OPT: Occupied Palestinian Territories. UAE: United Arab Emirates. The numbers vary by each variable due to missing values. * ${ }^{*}$-values for all types of tobacco use were derived from chi-squared tests. 
gender gap (7.7\%) in comparison to Jordan, the OPT, and the UAE. Overall, the prevalence of electronic cigarette use was low among students and more prevalent among males than females, except for Egypt where the prevalence among females was higher than among males and almost equal to that of male students in the UAE (Table 2).

Overall, $10.2 \%$ of the females ever smoked cigars compared to $23.7 \%$ of males $(p<0.001)$. Lower proportions were noted for regular pipe smoking $(8 \%$ and $12.3 \%$, respectively). Of the female students, $7.3 \%$ ever smoked midwakh compared to $17.4 \%$ of the males $(\mathrm{p}<0.001)$. UAE males had the highest prevalence of midwakh ever smokers (58\%) and current smokers $(52 \%)$ as well as the UAE females $(28.9 \%$ and $13.2 \%$, respectively). Smokeless tobacco was not commonly used by both sexes, with the lowest prevalence noted among females and males in the OPT (Table 2).

\section{First waterpipe smoking}

Over two-thirds of males and females smoked their first waterpipe when they were between the ages of 15 and 19 years (Table 3). The OPT had the highest proportion of males $(21.0 \%)$ who smoked their first waterpipe in the younger age group (10-14 years) and Egypt the highest proportion (18.5\%) among females.

Females used the waterpipe for the first time mostly with family members $(37.4 \%)$ and friends $(32.6 \%)$ while males initiated WTS with a friend or several

Table 3. Waterpipe smoking initiation in four EMR countries, $2016(\mathrm{~N}=2470)$

\begin{tabular}{|c|c|c|c|c|c|c|c|c|c|c|}
\hline \multirow[t]{2}{*}{ Initiation } & \multicolumn{2}{|c|}{ Jordan } & \multicolumn{2}{|c|}{ OPT } & \multicolumn{2}{|c|}{ UAE } & \multicolumn{2}{|c|}{ Egypt } & \multicolumn{2}{|c|}{ Total } \\
\hline & $\begin{array}{c}\text { Female } \\
n(\%)\end{array}$ & $\begin{array}{l}\text { Male } \\
n(\%)\end{array}$ & $\begin{array}{c}\text { Female } \\
n(\%)\end{array}$ & $\begin{array}{l}\text { Male } \\
n(\%)\end{array}$ & $\begin{array}{c}\text { Female } \\
n(\%)\end{array}$ & $\begin{array}{l}\text { Male } \\
n(\%)\end{array}$ & $\begin{array}{l}\text { Female } \\
n(\%)\end{array}$ & $\begin{array}{l}\text { Male } \\
n(\%)\end{array}$ & $\begin{array}{c}\text { Female } \\
n(\%)\end{array}$ & $\begin{array}{l}\text { Male } \\
n(\%)\end{array}$ \\
\hline \multicolumn{11}{|l|}{$\begin{array}{l}\text { Age at } \\
\text { waterpipe } \\
\text { smoking } \\
\text { initiation (years) }\end{array}$} \\
\hline $10-14$ & $22(8.0)$ & $53(11.4)$ & $41(11.2)$ & $72(21.0)$ & $8(10.8)$ & $13(14.1)$ & $12(18.5)$ & $51(9.9)$ & $83(10.6)$ & $189(13.4)$ \\
\hline $15-19$ & $195(70.7)$ & 365 (78.3) & $262(71.8)$ & $240(70.0)$ & $50(67.6)$ & $74(80.4)$ & $20(30.8)$ & $270(52.6)$ & $527(67.6)$ & $949(67.1)$ \\
\hline$\geq 20$ & $59(21.4)$ & $48(10.3)$ & $62(17.0)$ & $31(9.0)$ & $16(21.6)$ & $5(5.4)$ & $33(50.8)$ & $192(37.4)$ & $170(21.8)$ & 276 (19.5) \\
\hline Total & 276 & 466 & 365 & 343 & 74 & 92 & 65 & 513 & 780 & 1414 \\
\hline$p$-value ${ }^{*}$ & \multicolumn{2}{|c|}{$<0.001$} & \multicolumn{2}{|c|}{$<0.001$} & \multicolumn{2}{|c|}{0.008} & \multicolumn{2}{|c|}{0.003} & \multicolumn{2}{|c|}{0.116} \\
\hline \multicolumn{11}{|l|}{$\begin{array}{l}\text { People with } \\
\text { whom smoked } \\
\text { first waterpipe }\end{array}$} \\
\hline No one & $16(5.9)$ & $32(6.9)$ & $11(3.0)$ & $24(6.9)$ & $0(0.0)$ & $6(6.4)$ & $10(15.4)$ & 88 (17.7) & $37(4.8)$ & 150 (10.7) \\
\hline A friend & 79 (28.9) & $167(36.0)$ & $66(18.1)$ & $150(43.2)$ & $20(26.3)$ & $35(37.2)$ & 31 (47.7) & $228(46.0)$ & $196(25.2)$ & $580(41.4)$ \\
\hline Several friends & 91 (33.3) & $214(46.1)$ & 104 (28.6) & 135 (38.9) & $36(47.4)$ & $48(51.1)$ & $23(35.4)$ & $176(35.5)$ & $254(32.6)$ & 573 (40.9) \\
\hline Family members & 87 (31.9) & $51(11.0)$ & $183(50.3)$ & $38(11.0)$ & $20(26.3)$ & $5(5.3)$ & $1(1.5)$ & $4(0.8)$ & 291 (37.4) & $98(7.0)$ \\
\hline Total & 273 & 464 & 364 & 347 & 76 & 94 & 65 & 496 & 778 & 1401 \\
\hline$p$-value & \multicolumn{2}{|c|}{$<0.001$} & \multicolumn{2}{|c|}{$<0.001$} & \multicolumn{2}{|c|}{$<0.001$} & \multicolumn{2}{|c|}{0.904} & \multicolumn{2}{|c|}{$<0.001$} \\
\hline \multicolumn{11}{|l|}{$\begin{array}{l}\text { Place smoked } \\
\text { first waterpipe }\end{array}$} \\
\hline $\begin{array}{l}\text { Café/restaurant/ } \\
\text { smoke shop }\end{array}$ & $122(45.0)$ & $259(57.7)$ & 135 (35.9) & $143(45.1)$ & 53 (71.6) & $80(87.0)$ & $56(86.2)$ & 426 (84.9) & 366 (46.6) & $908(66.8)$ \\
\hline Own home & 77 (28.4) & 71 (15.8) & $168(44.7)$ & 71 (22.4) & $12(16.2)$ & $5(5.4)$ & $3(4.6)$ & 15 (3.0) & $260(33.1)$ & 162 (11.9) \\
\hline $\begin{array}{l}\text { Someone else's } \\
\text { home }\end{array}$ & $68(25.1)$ & $117(26.1)$ & 68 (18.1) & $90(28.4)$ & 8 (10.8) & 7 (7.6) & $4(6.2)$ & 46 (9.2) & $148(18.8)$ & 260 (19.1) \\
\hline $\begin{array}{l}\text { University } \\
\text { accommodation }\end{array}$ & $4(1.5)$ & $2(0.4)$ & $5(1.3)$ & $13(4.1)$ & $1(1.4)$ & $0(0.0)$ & $2(3.1)$ & $15(3.0)$ & $12(1.5)$ & $30(2.2)$ \\
\hline Total & 271 & 449 & 376 & 317 & 74 & 92 & 65 & 502 & 786 & 1360 \\
\hline p-value & \multicolumn{2}{|c|}{$<0.001$} & \multicolumn{2}{|c|}{$<0.001$} & \multicolumn{2}{|c|}{0.056} & \multicolumn{2}{|c|}{0.781} & \multicolumn{2}{|c|}{$<0.001$} \\
\hline
\end{tabular}

OPT: Occupied Palestinian Territories. UAE: United Arab Emirates. The numbers vary by each variable due to missing values. ${ }^{*}$-values for all ways of initiation were derived from chi-squared tests. 
friends $(82.3 \%)$. In all countries, the proportion of female students smoking their first waterpipe with family members exceeded that of males with the highest in the OPT $(50.3 \%)$. The majority of males (66.8\%) and females (46.6\%) smoked their first waterpipe in a café/restaurant with the highest proportion observed in Egypt (84.9\% and 86.2\%, respectively). Higher proportions of females smoked their first waterpipe at home $(33.1 \%)$ than males (11.9\%) with the highest being among female students in the OPT.

\section{Usual persons smoked waterpipe with and usual places of purchase}

Overall, $14 \%$ of females almost always/always smoked the waterpipe with family members compared to $3.3 \%$ of males $(\mathrm{p}<0.001)$, with the highest among females in Jordan (17.4\%) and the OPT (15.2\%). High proportions of females (45.0\%) and males $(38.3 \%)$ in Egypt almost always/always smoked alone compared to other countries. In addition, about one-third of females usually smoked waterpipe in a café/restaurant compared to $45 \%$ of males. The percentage of females who almost always/always smoked in a café/restaurant was similar to that of males within each country, with students in Egypt having the highest proportions for both females $(76.2 \%)$ and males $(77.0 \%)$. There were statistically significant differences $(p<0.001)$ between males and females with respect to the usual place of purchase of waterpipe tobacco, except in Egypt. More females $(38.9 \%)$ than males $(25.8 \%)$ did not purchase the waterpipe products they used, with UAE females and males having the highest proportions of not purchasing the products (Supplementary file, Table S1).

Eleven per cent of male students smoked nonflavored tobacco compared to $1 \%$ of females $(\mathrm{p}<0.001)$. In Egypt, $27.7 \%$ of the male students smoked nonflavored tobacco compared to $8.8 \%$ of the females $(\mathrm{p}=0.001)$. There was a positive strong correlation $(\mathrm{r}=0.808)$ between males and females by the top five ranked waterpipe flavors smoked $(\mathrm{p}<0.0001)$. The correlation was also strong for Jordan $(\mathrm{r}=0.911)$, the OPT $(r=0.890)$ and Egypt $(r=0.802)$ but moderate for the UAE ( $\mathrm{r}=0.468)$. Table 4 shows the top five waterpipe flavors by country. Apple/double apple, mint, grape, watermelon, and bubblegum, ranked

Table 4. Top five waterpipe flavors usually smoked by country and gender in four EMR countries, 2016 (N=2470)

\begin{tabular}{|c|c|c|c|c|c|c|c|c|c|}
\hline \multicolumn{2}{|c|}{ Jordan } & \multicolumn{2}{|c|}{ OPT } & \multicolumn{2}{|c|}{ UAE } & \multicolumn{2}{|c|}{ Egypt } & \multicolumn{2}{|c|}{ Total } \\
\hline $\begin{array}{c}\text { Female } \\
(\%)\end{array}$ & $\begin{array}{l}\text { Male } \\
(\%)\end{array}$ & $\begin{array}{c}\text { Female } \\
(\%)\end{array}$ & $\begin{array}{l}\text { Male } \\
(\%)\end{array}$ & $\begin{array}{c}\text { Female } \\
(\%)\end{array}$ & $\begin{array}{l}\text { Male } \\
(\%)\end{array}$ & $\begin{array}{c}\text { Female } \\
(\%)\end{array}$ & $\begin{array}{l}\text { Male } \\
(\%)\end{array}$ & $\begin{array}{c}\text { Female } \\
(\%)\end{array}$ & $\begin{array}{l}\text { Male } \\
(\%)\end{array}$ \\
\hline $\begin{array}{l}\text { Lemon mint } \\
\qquad(35.6)\end{array}$ & $\begin{array}{c}\text { Apple/ } \\
\text { double } \\
\text { apple } \\
(58.6)\end{array}$ & $\begin{array}{c}\text { Apple/ } \\
\text { double } \\
\text { apple } \\
(37.6)\end{array}$ & $\begin{array}{c}\text { Apple/ } \\
\text { double } \\
\text { apple } \\
(67.8)\end{array}$ & $\begin{array}{c}\text { Grape mint } \\
(49.4)\end{array}$ & $\begin{array}{c}\text { Apple/ } \\
\text { double } \\
\text { apple } \\
(41.2)\end{array}$ & $\begin{array}{c}\text { Apple/ } \\
\text { double } \\
\text { apple } \\
(48.5)\end{array}$ & $\begin{array}{c}\text { Apple/ } \\
\text { double } \\
\text { apple } \\
(46.0)\end{array}$ & $\begin{array}{l}\text { Apple/ } \\
\text { double } \\
\text { apple } \\
(36.4)\end{array}$ & $\begin{array}{c}\text { Apple/ } \\
\text { double } \\
\text { apple } \\
(55.2)\end{array}$ \\
\hline $\begin{array}{c}\text { Apple/ } \\
\text { double } \\
\text { apple } \\
(33.2)\end{array}$ & $\begin{array}{c}\text { Lemon mint } \\
(23.7)\end{array}$ & $\begin{array}{c}\text { Watermelon } \\
\text { mint } \\
(35.8)\end{array}$ & $\begin{array}{c}\text { Lemon mint } \\
(25.9)\end{array}$ & $\begin{array}{l}\text { Mint } \\
(45.5)\end{array}$ & $\begin{array}{c}\text { Grape mint } \\
(40.2)\end{array}$ & $\begin{array}{c}\text { Watermelon } \\
(25.0)\end{array}$ & $\begin{array}{c}\text { Non- } \\
\text { flavored } \\
(27.7)\end{array}$ & $\begin{array}{l}\text { Mint } \\
(16.8)\end{array}$ & $\begin{array}{l}\text { Grape } \\
(14.2)\end{array}$ \\
\hline $\begin{array}{c}\text { Blueberry } \\
(25.3)\end{array}$ & $\begin{array}{c}\text { Watermelon } \\
\text { mint } \\
(20.5)\end{array}$ & $\begin{array}{c}\text { Lemon mint } \\
(34.8)\end{array}$ & $\begin{array}{c}\text { Watermelon } \\
\text { mint } \\
(25.4)\end{array}$ & $\begin{array}{c}\text { Apple/ } \\
\text { double } \\
\text { apple } \\
(31.2)\end{array}$ & $\begin{array}{l}\text { Mint } \\
(27.5)\end{array}$ & $\begin{array}{c}\text { Lemon mint } \\
(13.2)\end{array}$ & $\begin{array}{c}\text { Lemon mint } \\
(23.1)\end{array}$ & $\begin{array}{l}\text { Grape } \\
(13.9)\end{array}$ & $\begin{array}{c}\text { Watermelon } \\
(11.6)\end{array}$ \\
\hline $\begin{array}{c}\text { Watermelon } \\
\text { mint } \\
(23.6)\end{array}$ & $\begin{array}{l}\text { Grape } \\
(15.9)\end{array}$ & $\begin{array}{c}\text { Mulberry } \\
(16.1)\end{array}$ & $\begin{array}{l}\text { Grape } \\
(10.3)\end{array}$ & $\begin{array}{l}\text { Grape } \\
(28.6)\end{array}$ & $\begin{array}{l}\text { Grape } \\
(24.5)\end{array}$ & $\begin{array}{c}\text { Chocolate, } \\
\text { lemon, } \\
\text { strawberry, } \\
\text { non- } \\
\text { flavored } \\
(8.8)\end{array}$ & $\begin{array}{l}\text { Grape } \\
(13.3)\end{array}$ & $\begin{array}{c}\text { Watermelon } \\
\text { (13.4) }\end{array}$ & $\begin{array}{l}\text { Mint } \\
(9.8)\end{array}$ \\
\hline $\begin{array}{l}\text { Bubble gum } \\
\qquad(19.5)\end{array}$ & $\begin{array}{c}\text { Blueberry } \\
\text { (13.3) }\end{array}$ & $\begin{array}{c}\text { Cinnamon } \\
\text { gum } \\
(15.6)\end{array}$ & $\begin{array}{c}\text { Mulberry } \\
(10.0)\end{array}$ & $\begin{array}{c}\text { Watermelon } \\
\text { (20.8) }\end{array}$ & $\begin{array}{c}\text { Watermelon } \\
\text { (13.7) }\end{array}$ & $\begin{array}{l}\text { Grape } \\
(7.4)\end{array}$ & $\begin{array}{c}\text { Watermelon } \\
\text { (12.9) }\end{array}$ & $\begin{array}{l}\text { Bubble gum } \\
\text { (13.2) }\end{array}$ & $\begin{array}{c}\text { Bubble gum } \\
(9.3)\end{array}$ \\
\hline
\end{tabular}


among the top five flavors for both genders with some variation in ranking by country. Apple/double apple was the most popular flavor for both genders in the overall sample and all countries, with the exception of females from Jordan and the UAE.

\section{Most recent waterpipe smoking experience}

Table 5 shows that the duration of smoking in the most recent smoking session varied between genders across countries and was statistically significant, except in the OPT. Almost half of the females smoked waterpipe for $<0.5$ hours compared to $30.5 \%$ of the males $(\mathrm{p}<0.001)$. Over one-third $(34.1 \%)$ of males smoked $\geq 10$ heads compared to $14.9 \%$ of the females. The difference by gender was highly statistically significant $(p<0.001)$ overall, and in each country with the exception of the UAE. The majority of males (65.1\%) and females (48.3\%) in the overall sample had smoked their last waterpipe in a café/restaurant $(\mathrm{p}<0.001)$.

The mean price paid for the last smoking session by females $(7.58 \pm 4.48$ US $\$)$ across all countries was higher than that of males $(4.68 \pm 4.62$ US $\$)$. In individual countries, females reported paying more than their male counterparts with differences that were statistically significant except for the UAE which

Table 5. Most recent waterpipe tobacco smoking session by duration of smoking, number of waterpipe heads, and place smoked in four EMR countries, 2016 ( $\mathrm{N}=2470)$

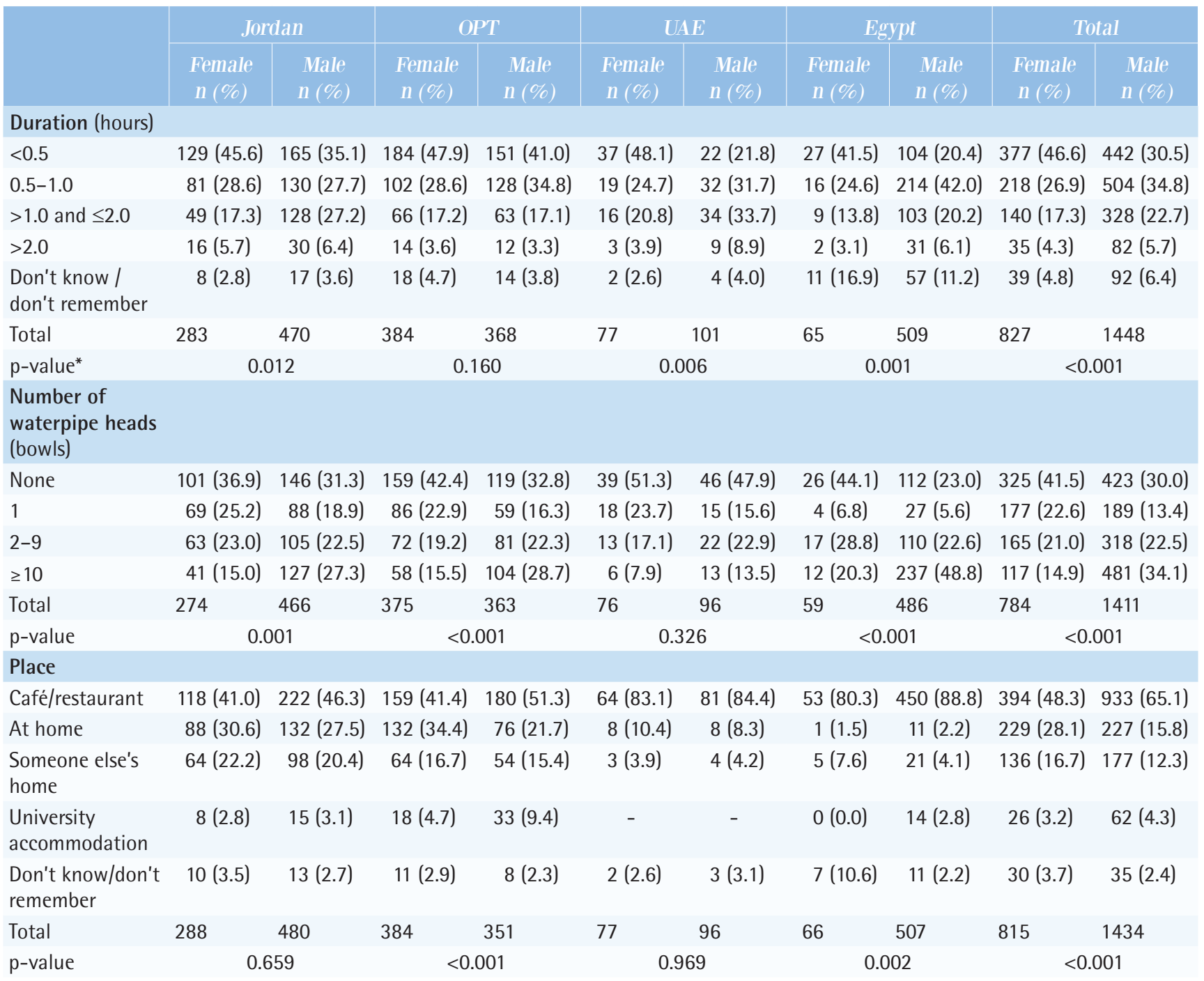

OPT: Occupied Palestinian Territories. UAE: United Arab Emirates. The numbers vary by each variable due to missing values. ${ }^{*} p$-values for each waterpipe factor were derived from chi-squared tests. 
had the highest price paid by females $(14.05 \pm 5.93$ US\$) and males $(12.63 \pm 8.16$ US\$).

\section{DISCUSSION}

Although the gender gap in smoking prevalence is narrowing in several countries ${ }^{17}$, the gap is generally wide in countries of the EMR. However, recent studies indicate that the gender gap is narrowing for certain tobacco products ${ }^{5}$. The increasing social acceptability of female smoking in the region, the perception of reduced harm with flavored waterpipe tobacco in comparison to cigarettes and its wide accessibility are among the reasons behind the narrowing of the gap $^{4,5,7,8}$.

The EMR has the highest prevalence of WTS globally, with a higher prevalence of WTS among males than females ${ }^{18}$. One in ten youth in the EMR smoke waterpipe, with variation between countries and a higher prevalence among males ${ }^{3}$. In this study, the gender gap for WTS was much lower than that of cigarette smoking. This is in accordance with previous studies from the region that reported a narrower gap for waterpipe than cigarettes ${ }^{2,5,6}$. As for electronic cigarettes and electronic waterpipe, the gender gap was similar. Recent evidence from Egypt indicates that ENDS use is higher among females and the gender gap in certain types of tobacco use is narrowing ${ }^{5}$. The gender differences with respect to age, educational level, marital status and employment might have affected the gap by making it narrower than it would have been if these differences did not exist.

Waterpipe tobacco consumption was also higher among males than females in our study. This in support to what has been reported from Jordan where the proportion of male $(40.6 \%)$ dental university students, who smoked more than two sessions per week, was more than double that of females $(18.6 \%)^{19}$.

Midwakh smoking was not common among the study participants except among UAE males and females. A recent study on adult UAE nationals reported that midwakh was the second most common type of smoking with a prevalence of $3.6 \%$ among males and rarely smoked by females ${ }^{20}$. Another UAE study on youth aged $13-15$ years ${ }^{21}$ reported a 9.7\% prevalence among males, double that of females $(3.4 \%)$, while a lower prevalence $(6.7 \%$ and $2.7 \%$, respectively) was reported from Lebanon ${ }^{22}$ among youth aged 12-18 years. Smokeless tobacco was uncommon among waterpipe smokers in our study. This in line with what has been reported from most EMR countries, particularly among youth ${ }^{23}$. Smokeless tobacco is however used in Sudan and Yemen particularly among males and to a lesser extent in Egypt and Saudi Arabia ${ }^{24}$.

Although the majority of males and females in our study started smoking at the ages of 15-19 years, the age group is older than what has been reported for schoolchildren in a study from Jordan where $58 \%$ of males and $63 \%$ of females started to smoke between the ages of 11 and 14 years $^{25}$. It is, however, similar to what has been reported by dental university students in Jordan ${ }^{19}$ where half started waterpipe smoking between 16 and 18 years.

Across all countries, the proportion of female students smoking their first waterpipe with family exceeded that of males. It is not surprising that over one-third of females started WTS with family members, which indicates the social acceptability of WTS among females in the Arab culture. The fact that half of females from the OPT smoked their first waterpipe with family members, in contrast to $1.5 \%$ of females from Egypt, shows variation in social acceptability of female WTS in countries of the region ${ }^{4}$. The study of dental students from Jordan found that almost half of female students smoked waterpipe in their parents' presence in contrast to $21.7 \%$ of males ${ }^{19}$. WTS by females in front of their parents, relatives and in public can be attributed to the social acceptability of its use in contrast to the negative attitude towards cigarette smoking by women ${ }^{4}$. Studies from Iran, an EMR non-Arab country but with similar culture and traditions to Arab countries, have underscored the role of the family in WTS initiation ${ }^{26}$. Having higher proportions in both sexes of always smoking alone in Egypt is worrying as a recent study from Egypt reported it as an independent determinant of selfreported addiction to $\mathrm{WTS}^{27}$.

The fact that females paid more than males per tobacco session could be partly attributed to the increasing power of spending among women ${ }^{13}$ and that females do not usually go to traditional cafés that generally charge less and are mainly frequented by men.

It has been reported that women usually prefer sweet waterpipe tobacco flavors like fruits, candy 
and chocolate instead of other flavors and unflavored tobacco ${ }^{28}$. Moreover, flavored waterpipe tobacco is more popular among the young as reported from the US and the Arab region ${ }^{12,15,29}$. Hence, it is not surprising that only $1 \%$ of females usually smoked non-flavored tobacco in our study compared to $11 \%$ of their male counterparts. Previous studies from the region affirmed that females prefer flavored tobacco for its attractive taste and smell ${ }^{30}$ that motivate many to start and continue WTS ${ }^{31,32}$. Flavored waterpipe tobacco has abundant flavors, with 'two apples' being the most popular ${ }^{32}$. In our study, the ranks of the flavors were highly correlated between genders. Apple/double apple was the most popular flavor smoked among respondents of both sexes in the total sample, in the OPT and Egypt, and among males in Jordan and the UAE. Although not much is yet known about the potential health risks of flavored tobacco $^{32}$, the need to have regulations to restrict flavors in waterpipe tobacco similar to cigarettes has been raised $^{31}$.

The current study showed that the gender gap is narrowing with respect to the prevalence of WTS and age of initiation of WTS in the EMR. In addition, the socio-cultural deterrents of WTS uptake and continued smoking are diminishing for youth in both genders. Equal opportunities are given to the young in both sexes by their families and communities in our region to smoke the waterpipe. This WTS encouraging environment for females, the available varieties of flavors, the flourishing café culture and the tobacco marketing strategies that target women puts women in the EMR on equal grounds with their male peers ${ }^{12,23,24,33}$. Women in the EMR seem to have similar freedom as males in accessing waterpipe tobacco products and in smoking at home or outside. While Goal 5 of the Sustainable Development Goals (SDG5) calls for achieving gender equality, empowering women and abolishing discrimination against them ${ }^{34}$, it is not the intention of this goal to increase their access to harmful tobacco products but to provide knowledge on the WTS hazards equally to both genders ${ }^{35}$.

The results of our study have implications for educational establishments, tobacco control organizations and women societies to raise awareness that WTS is not a socially acceptable behavior and not to only educate them on its harms. Further, health policy makers are urged to control the expansion of the waterpipe serving establishments and ban use of flavors in tobacco products including waterpipe.

\section{Limitations}

Our study has some limitations as it included four EMR countries and six universities, which makes it difficult to generalize to all EMR countries and university students. Further, the number of study participants varied by country and we were unable to reach the target number of students in the UAE; however, this study provides unique insights into waterpipe patterns among young adults in four EMR countries, including in the UAE. Another limitation is that the study was based on convenience sampling. However, our study is one of the few studies that have examined WTS and gender, and assessed several related factors in the region. Moreover, the use of a web survey could have increased the participation rate of females in the survey and the reliability of their answers.

\section{CONCLUSIONS}

Male students had higher prevalence than females of all forms of tobacco smoking with a narrower gender gap for WTS. There were gender differences with respect to WTS in several aspects: age of initiation, person(s) with whom and place at which first smoked WTS, duration of smoking, tobacco consumption, place of purchase, and expenditure. The ease of accessibility of waterpipe tobacco from homes and cafés in the region make it easier for the young, and females in particular, to start smoking and maintain this habit. Further, the availability of different tobacco flavors in countries of the region calls for action as it motivates the young to start smoking, particularly females. Regular surveillance of the prevalence of WTS is needed to monitor the gender gap.

\section{REFERENCES}

1. Jawad M, Lee JT, Millett C. Waterpipe Tobacco Smoking Prevalence and Correlates in 25 Eastern Mediterranean and Eastern European Countries: Cross-Sectional Analysis of the Global Youth Tobacco Survey. Nicotine Tob Res. 2016;18(4):395-402. doi:10.1093/ntr/ntv101

2. Khattab A, Javaid A, Iraqi G, et al. Smoking habits in the Middle East and North Africa: results of the BREATHE study. Respir Med. 2012;106 Suppl 2:S16-S24. doi:10.1016/S0954-6111(12)70011-2 
3. Kheirallah KA, Alsulaiman JW, Mohammad HAS, Alzyoud S, Veeranki SP, Ward KD. Waterpipe Tobacco Smoking among Arab Youth; a Cross-Country Study. Ethn Dis. 2016;26(1):107-112. doi:10.18865/ed.26.1.107

4. Khalil J, Afifi R, Fouad FM, et al. Women and waterpipe tobacco smoking in the eastern mediterranean region: allure or offensiveness. Women Health. 2013;53(1):100116. doi:10.1080/03630242.2012.753978

5. Mostafa A, El Houssinie M, Fotouh AA. Multiple tobacco use among young adult waterpipe smokers in Egypt. East Mediterr Health J. 2018;24(1):7-17. doi:10.26719/2018.24.1.17

6. Tucktuck M, Ghandour R, Abu-Rmeileh NME. Waterpipe and cigarette tobacco smoking among Palestinian university students: a cross-sectional study. BMC Public Health. 2017;18(1). doi:10.1186/s12889-017-4524-0

7. Akl EA, Jawad M, Lam WY, Co CN, Obeid R, Irani J. Motives, beliefs and attitudes towards waterpipe tobacco smoking: a systematic review. Harm Reduct J. 2013;10:12. doi:10.1186/1477-7517-10-12

8. Afifi R, Khalil J, Fouad F, et al. Social norms and attitudes linked to waterpipe use in the Eastern Mediterranean Region. Soc Sci Med. 2013;98:125-134. doi:10.1016/j.socscimed.2013.09.007

9. Salloum RG, Abu-Rmeileh N, Hamadeh R, et al. PolicyRelevant Context of Waterpipe Tobacco Smoking among University Students in Six Countries Across the Eastern Mediterranean Region: A Qualitative Study. Asian Pac J Cancer Prev. 2017;18(9):2533-2540. doi:10.22034/APJCP.2017.18.9.2533

10. Poyrazoğlu S, Sarli S, Gencer Z, Günay O. Waterpipe (narghile) smoking among medical and non-medical university students in Turkey. Ups J Med Sci. 2010;115(3):210-216. doi:10.310 9/03009734.2010.487164

11. Salloum RG, Maziak W, Hammond D, et al. Eliciting preferences for waterpipe tobacco smoking using a discrete choice experiment: implications for product regulation. BMJ Open. 2015;5:e009497. doi:10.1136/bmjopen-2015-009497

12. Mostafa A, Mohammed HT, Hussein WM, et al. Plain packaging of waterpipe tobacco? A qualitative analysis exploring waterpipe smokers' and non-smokers' responses to enhanced versus existing pictorial health warnings in Egypt. BMJ Open. 2018;8(10):e023496. doi:10.1136/bmjopen-2018-023496

13. World Health Organization. Gender, women, and the tobacco epidemic. World Health Organization; 2010 . https://apps.who.int/iris/bitstream/ handle/10665/44342/9789241599511_engpdf;jsessio nid=EAF2 770EDEE0D EF446262ECF4AD9A209? sequence=1. Accessed July 26, 2020.

14. Abu-Rmeileh N, Alkhuffash O, Kheirallah K, et al. Harm perceptions of waterpipe tobacco smoking among university students in five Eastern Mediterranean Region countries. Tob Induc Dis. 2018;16(May). doi:10.18332/tid/89966

15. Salloum RG, Nakkash R, Abu-Rmeileh NME, et al. Individual-level determinants of waterpipe smoking demand in four Eastern-Mediterranean countries. Health Promot Int. 2019;34(6):1157-1166. doi:10.1093/heapro/day084

16. Salloum RG, Lee JH, Mostafa A, et al. Waterpipe Tobacco Smoking among University Students in Three Eastern Mediterranean Countries: Patterns, Place, and Price. Subst Use Misuse. 2019;54(14):2275-2283. doi:10.1080/10826084.2019.1645177

17. Flandorfer P, Wegner-Siegmundt C, Buber I. Gender roles and smoking behaviour. Vienna Institute of Demography Working Papers. Vienna Institute of Demography; 2010:125. https://www.oeaw.ac.at/fileadmin/subsites/Institute/ VID/PDF/Publications/Working_Papers/WP2010_07. pdf. Published July, 2010. Accessed July 26, 2020.

18. WHO Framework Convention on Tobacco Control. Control and prevention of waterpipe tobacco products. World Health Organization; 2016. https://www.who.int/ fctc/cop/cop7/FCTC_COP_7_10_EN.pdf. Accessed May $29,2020$.

19. Obeidat SR, Khabour OF, Alzoubi KH, et al. Prevalence, social acceptance, and awareness of waterpipe smoking among dental university students: a cross sectional survey conducted in Jordan. BMC Res Notes. 2014;7:832. doi:10.1186/1756-0500-7-832

20. Al-Houqani M, Ali R, Hajat C. Tobacco smoking using Midwakh is an emerging health problem-evidence from a large cross-sectional survey in the United Arab Emirates. PloS One. 2012;7(6):e39189. doi:10.1371/journal.pone.0039189

21. Jawad M, Al-Houqani M, Ali R, et al. Prevalence, attitudes, behaviours and policy evaluation of midwakh smoking among young people in the United Arab Emirates: Cross-sectional analysis of the Global Youth Tobacco Survey. PloS One. 2019;14(4):e0215899. doi:10.1371/journal.pone.0215899

22. Afifi R, Saravanan M, El Salibi N, et al. Evidence from the Lebanon Global School-based Student Health Survey on midwakh tobacco smoking in school students: a harbinger of the next global tobacco pandemic? East Mediterr Health J. 2020;26(1):116-121. doi:10.26719/2020.26.1.116

23. Maziak W, Nakkash R, Bahelah R, Husseini A, Fanous N, Eissenberg T. Tobacco in the Arab world: old and new epidemics amidst policy paralysis. Health Policy Plan. 2014;29(6):784-794. doi:10.1093/heapol/czt055

24. Drope J, Schluger N, Gahn Z, et al. The Tobacco Atlas. 6th ed. American Cancer Society; 2018.

25. McKelvey KL, Wilcox ML, Madhivanan P, Mzayek F, Khader YS, Maziak W. Time trends of cigarette and waterpipe smoking among a cohort of school children in Irbid, Jordan, 2008-11. Eur J Public Health. 2013;23(5):862-867. doi:10.1093/eurpub/ckt140

26. Baheiraei A, Shahbazi Sighaldeh S, Ebadi A, Kelishadi R, Majdzadeh R. The Role of Family on Hookah Smoking 
Initiation in Women: A Qualitative Study. Glob J Health Sci. 2015;7(5):1-10. doi:10.5539/gjhs.v7n5p1

27. Mostafa A. Self-reported addiction to and perceived behavioural control of waterpipe tobacco smoking and its patterns in Egypt: policy implications. East Mediterr Health J. 2020;26(1):18-28. doi:10.26719/2020.26.1.18

28. Scott-Sheldon LAJ, Stroud LR. Preferences and Perceptions of Flavored Hookah Tobacco among US Women. Am J Health Behav. 2018;42(3):37-46. doi:10.5993/AJHB.42.3.4

29. Nakkash RT, Khalil J, Afifi RA. The rise in narghile (shisha, hookah) waterpipe tobacco smoking: a qualitative study of perceptions of smokers and non smokers. BMC Public Health. 2011;11:315. doi:10.1186/1471-2458-11-315

30. Abdulrashid OA, Balbaid O, Ibrahim A, Shah HBU. Factors contributing to the upsurge of water-pipe tobacco smoking among Saudi females in selected Jeddah cafes and restaurants: A mixed method study. J Family Community Med. 2018;25(1):13-19. doi:10.4103/jfcm.JFCM_3_17

31. Leavens EL, Driskill LM, Molina N, et al. Comparison of a preferred versus non-preferred waterpipe tobacco flavour: subjective experience, smoking behaviour and toxicant exposure. Tob Control. 2018;27:319-324. doi:10.1136/tobaccocontrol-2016-053344

32. Schubert J, Luch A, Schulz TG. Waterpipe smoking: analysis of the aroma profile of flavored waterpipe tobaccos. Talanta. 2013;115:665-674. doi:10.1016/j.talanta.2013.06.022

33. Gilmore AB, Fooks G, Drope J, Bialous SA, Jackson RR. Exposing and addressing tobacco industry conduct in low-income and middle-income countries. Lancet. 2015;385(9972):1029-1043. doi:10.1016/S0140-6736(15)60312-9

34. United Nations Development Programme. Sustainable Development Goals. https://sdgs.un.org/goals. Accessed June 15, 2020.

35. Hamadeh RR, Ahmed J, Jassim GA, Alqallaf SM, AlRoomi K. Knowledge of health professional students on waterpipe tobacco smoking: curricula implications. BMC Med Educ. 2018;18:300. doi:10.1186/s12909-018-1406-9
ACKNOWLEDGEMENTS

The authors acknowledge the following individuals and research teams for their contributions to the data collection phase of this study: Mostafa Elshahed and Marwa Nofal on behalf of the Medical Students Awareness Group 'Arabicrosheta' (Ain Shams University, Egypt); Rula Ghandour and Marina Touktouk (Birzeit University, Occupied Palestinian Territories); and the International Federation of Medical Students Association-Jordan/Jordan University of Science and Technology Local community (IFMSA-JO/JUST-Lc), as well as Gray Babbs at the Arabian Gulf University, Bahrain.

\section{CONFLICTS OF INTEREST}

The authors have completed and submitted the ICMJE Form for Disclosure of Potential Conflicts of Interest and none was reported.

\section{FUNDING}

This work was supported by the International Development Research Centre [Grant reference number 106981-001, Ottawa, Canada (https:// www.idrc.ca)]. The funding source had no involvement in the study design, collection, analysis, and interpretation of the data, in writing the manuscript, and in deciding to submit it for publication.

\section{AUTHORS' CONTRIBUTIONS}

RRH made substantial contributions to the conception and design of the study, analysis, and interpretation of data, writing the first draft of the manuscript and revising it critically for important intellectual content, gave final approval of the submitted version to be published and is accountable for all aspects of the work. JL performed the analysis. NMEAR, MD, AM, KAK, AY, JT and MS contributed in supervising the acquisition of data. RN solicited the funds, and contributed to the conception and design of the study. RGS contributed to the conception and design of the study and in the analysis. All authors commented on previous versions of the manuscript and approved the final version for publication.

PROVENANCE AND PEER REVIEW

Not commissioned; externally peer reviewed. 